\title{
Resonant Motions of Dynamic Offshore Structures in Large Waves
}

\author{
Ove Tobias Gudmestad
}

Citation: Gudmestad, O.T. Resonant Motions of Dynamic Offshore Structures in Large Waves. Fluids 2021, 6, 352. https://doi.org/ $10.3390 /$ fluids 6100352

Academic Editors: Sheldon Wang and Mehrdad Massoudi

Received: 22 July 2021

Accepted: 30 September 2021

Published: 7 October 2021

Publisher's Note: MDPI stays neutral with regard to jurisdictional claims in published maps and institutional affiliations.

Copyright: (C) 2021 by the author. Licensee MDPI, Basel, Switzerland. This article is an open access article distributed under the terms and conditions of the Creative Commons Attribution (CC BY) license (https:// creativecommons.org/licenses/by/ $4.0 /)$.
Department of Mechanical and Structural Engineering and Material Science, University of Stavanger, 4036 Stavanger, Norway; otgudmestad@gmail.com or ove.t.gudmestad@uis.no; Tel.: +47-48100259

\begin{abstract}
In marine engineering, the dynamics of fixed offshore structures (for oil and gas production or for wind turbines) are normally found by modelling of the motion by a classical mass-spring damped system. On slender offshore structures, the loading due to waves is normally calculated by applying a force which consists of two parts: a linear "inertia/mass force" and a non-linear "drag force" that is proportional to the square of the velocity of the particles in the wave, multiplied by the direction of the wave particle motion. This is the so-called Morison load model. The loading function can be expanded in a Fourier series, and the drag force contribution exhibits higher order harmonic loading terms, potentially in resonance with the natural frequencies of the system. Currents are implemented as constant velocity terms in the loading function. The paper highlights the motion of structures due to non-linear resonant motion in an offshore environment with high wave intensity. It is shown that "burst" / "ringing" type motions could be triggered by the drag force during resonance situations.
\end{abstract}

Keywords: resonant motions; one degree of freedom system dynamics; non-linear drag loading; limit cycles; ringing; higher order resonances

\section{Introduction}

The irregular wave environment is normally modelled as a linear sum of sinusoidal waves, using a Fourier representation of the actual wave environment. Actual waves could, however, exceed this representation, as "freak waves" / "rogue waves" cannot be explained by the statistics of irregular waves. Crests become higher (Stokes non-linear effects) and non-linear wave-wave interactions make the waves grow at the expense of others [1,2].

The basic equations of wave analysis are partial differential equations with bottom and surface boundary conditions, having an unlimited number of solutions. A particular class of solutions is the non-linear soliton waves that satisfy all equations of the hydrodynamics. Using this type of solution, we assume that, at low probability of exceedance, an irregular large sea state is not composed of independent linear waves. Data documenting large waves where the maximum wave height is larger than twice the significant wave $\left(2 \times \mathrm{H}_{\mathrm{s}}\right)$, the so-called freak or rogue waves, were initially presented by Sand et al. [3] and further documented by Haver [4,5]. Osborne [6] has extensively developed the theory for rogue waves. However, in the calculation of the response of structures to waves, the description of the loading is normally represented by sinusoidal waves; see Section 3.

The wave and current loading on structures which are "slender" in comparison with the wavelength are normally calculated with the use of the so-called "Morison equation" [7-11]. For structures where the diameter to wave-height ratio is larger than 0.1 , both the drag and the inertia (mass) terms should be taken into account, and the drag term's contribution to the maximum loading is reduced as the diameter increases. While the mass term represents a linear function of the wave-height, the drag term represents a non-linear contribution to the loading; it is of interest to investigate further the non-linear loading effects and higher order non-linear effects [12]. 
An assumption behind the use of the Morison equation is that the wave length is long compared to the diameter of the structural elements, so a linear combination of forces caused by a constant velocity term (drag loading) and the constant accelerating term (mass loading) will describe the motion caused by the oscillatory forces. In the case of slender structural cylindrical elements, where the diameter of the element, D, is less than $10 \%$ of the wave height, the drag term will dominate. When the diameter of the cylinder is in the order of half the wave height, both drag and mass terms will be important [11]. A thorough discussion of the relative values of the drag and the mass/inertia components is given in [8]. As it is of particular interest to study the response ( $x$ is the displacement of the structure as a function of time) to non-linear load effects (as these can give rise to higher order non-linear resonances [12]), a one degree of freedom system with a non-linear drag loading term of the Morison type, caused by a wave having a frequency, $\omega$, could be reformulated as:

$$
m \frac{d^{2} x}{d t^{2}}+c \frac{d x}{d t}+k x(t)=F_{0} \sin (\omega t)|\sin (\omega t)|
$$

Here, $F_{0}$ is a constant representing the height of the wave, $t$ is the time, and $m, c$ and $k$ represent the system's mass, damping and stiffness, respectively. The forcing term represents a term proportional to the wave particle velocity squared, representing turbulent flow past a cylinder (an obstacle in the flow path).

The main objective of this paper is to investigate resonance phenomena in a wavestructure interaction system where the wave loading frequency is a fraction $(1 / 2$ or $1 / 3)$ of the natural frequency of the structural system in the case of non-linear wave loading. The method of phase plane presentation will be used to identify limit cycles for certain combinations of initial values. A detailed study of the effects of the parameters of the non-linear forcing equation is presented in [13]. The main contribution presented in the present paper is to link the theoretical findings to a case example: the strong vibrations of a one-tower concrete platform located in the Norwegian Sea, when hit by a very large wave [14].

It should be noted that the response of a structure is often calculated in the time domain given the wave profile; however, analytical studies, like the study presented herein, help to identify specific aspects of the solution, like the large non-linear resonances: the "burst" / "ringing" effect reported in this paper. The drag and inertia coefficients should be given as functions of the water wave kinematics; however, often these coefficients are taken as constants over the water depth and over the wave field, as the main contributions to the loading come from near-surface effects in the largest waves [11].

\section{The Phase Plane and Limit Cycles}

For the further discussion in this paper, it is necessary to introduce the concepts of the phase plane and the limit cycle. For a system where the solution is time-dependent, the phase plane shows the displacement as a function of time on one axis (the $x$-axis) and the variation of the displacement (the velocity) on the other axis (the $y$-axis). The phase plane method is often used to investigate the solution of non-linear differential equations of importance in engineering applications. A linear second order ordinary differential equation with no external driving force will have a phase plane solution given by ellipsoids, while the solution when damping is introduced will spiral toward a point of equilibrium, a singular point. This is natural, as damping taps the system for motion energy.

The solution of non-linear differential equations (where the non-linearity could be in the stiffness, damping or loading terms) could move towards a point of equilibrium (as when damping is introduced), or the solution could move away from the initial point (given by the initial values of displacement and velocity). In this case, energy is transmitted to the system in the form of external energy transfer, or an apparent negative damping is introduced. In the case of the trajectories in the $(x(t), d x(t) / d t)$-plane spiralling towards or away from a closed trajectory, we call this trajectory a limit cycle [15]. In the case of the trajectories spiralling towards the limit cycle, the limit cycle is called a stable limit cycle. 
An unstable limit cycle is a limit cycle where the trajectories spiral away from the limit cycle. In this case, all initial points (at $t=0$ ) starting outside the limit cycle will spiral away, and we see an increase in displacement and velocity. Note that new limit cycles may be reached at another level of motion of the system. Initial points starting at values placing the starting point inside the limit cycle will lead to reduced motion; in most cases, the solution will tend towards the singular point where the motion stops. Systems where the limit cycle is stable represent those where the generation of energy is balanced with the energy dissipation (the damping). For more references regarding phase planes, limit cycles and systems where the generation and dissipation of energy are equal, see [16,17]. Note that limit cycles can occur only when non-linear terms are present; linear systems would have varying trajectories, depending on the initial conditions.

A second order ordinary differential equation often used to describe phenomena in electrical engineering and medicine [18] (for example, to explain tremors caused by Parkinson's disease) is the van der Pol Equation (2). The solution of this equation exhibits limit cycles.

$$
\frac{d^{2} x}{d t^{2}}-\mu\left(1-x^{2}\right) \frac{d x}{d t}+x(t)=0
$$

When starting with relatively large initial values for displacement and velocity, the solution will converge towards a limit cycle. In the case of the initial vales being located inside the limit cycle, the displacement and velocity of the motion will increase towards the limit cycle; the situation could be regarded as a negative damping term pumping energy into the system. The location of the limit cycle will depend on the value of the parameter $\mu$. Figure 1 shows the phase plane diagram for the van der Pol equation with $\mu=1$.0. The initial conditions are given by small squares in the figure. The arrows give the direction of the motion with increasing time. It is noted that all trajectories (whether we start from outside or inside the limit cycle) spiral towards the limit cycle. For further discussion of the van der Pol equation, see [17,19].

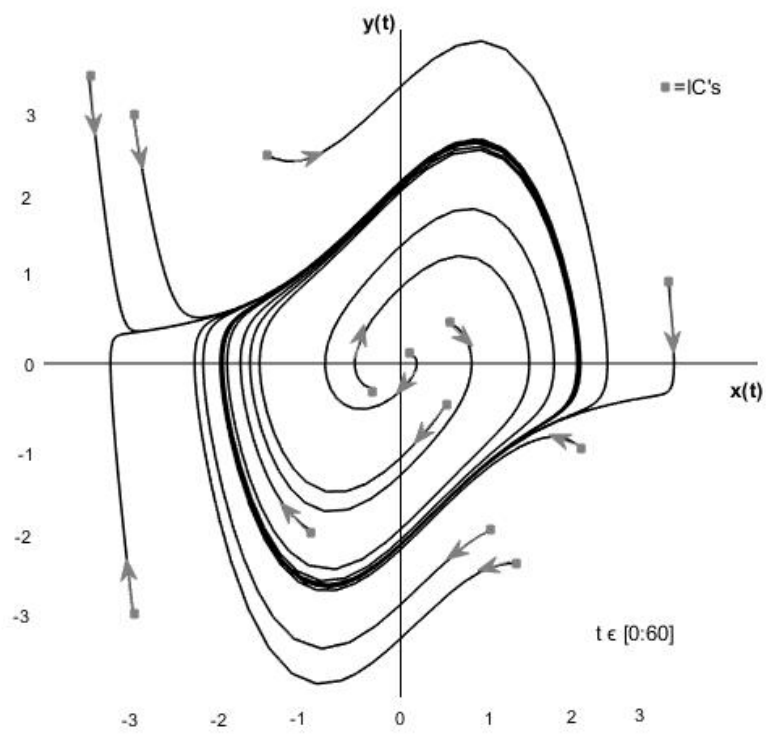

Figure 1. Phase plane diagram for the van der Pol equation with $\mu=1.0$ (and without forcing term) exhibiting a limit cycle, $v=d x / d t$. Reproduced from [13], Published by IOP Publishing 2017. 


\section{Drag Loading}

\subsection{Resonance}

The first order resonance between the loading and the system occurs when the frequency of the loading is equal to the frequency of the system, i.e., when $\omega=\omega_{0}$, where the system's natural frequency $\omega_{0}$ is given as:

$$
\omega_{0}=\sqrt{k / m}
$$

During the resonance, the energy from the wave is transferred to the system at the frequency of the system. The growth of the system's motion is limited by the damping, c, inherent in the system.

\subsection{Limit Cycles for System with Non-Linear Loading}

In paper [13], Equation (2) is considered, and the results of solutions are given for linear, constant damping, $c=0.5$.

The drag force damping term, $c(d x(t) / d t)$, is proportional to the velocity of the structural displacement, and the drag loading term is proportional to the wave water velocity squared. In [13], we investigated whether the drag forcing can act as a damping term in the system, and also whether it could act as negative damping, enhancing the motions of the structure. It is also of importance in this regard that the term $\sin (\omega t)|\sin (\omega t)|$ can be linearized using Fourier series transformation, whereby the wave forcing has terms with frequencies at integers of the wave frequency, $\omega$, potentially at higher order resonance frequencies:

$$
\sin (\omega t)|\sin (\omega t)|=c_{0}+c_{1} \sin (\omega t)+c_{2} \cos (2 \omega t)+c_{3} \sin (3 \omega t)+c_{4} \cos (4 \omega t)+\cdots
$$

It should be noted that large near-breaking waves have periods in the range of $15 \mathrm{~s}$, see Figure 2, which means that there is a third order resonance with structures having a natural period of $5 \mathrm{~s}$. Rogue waves could have periods which are shorter.

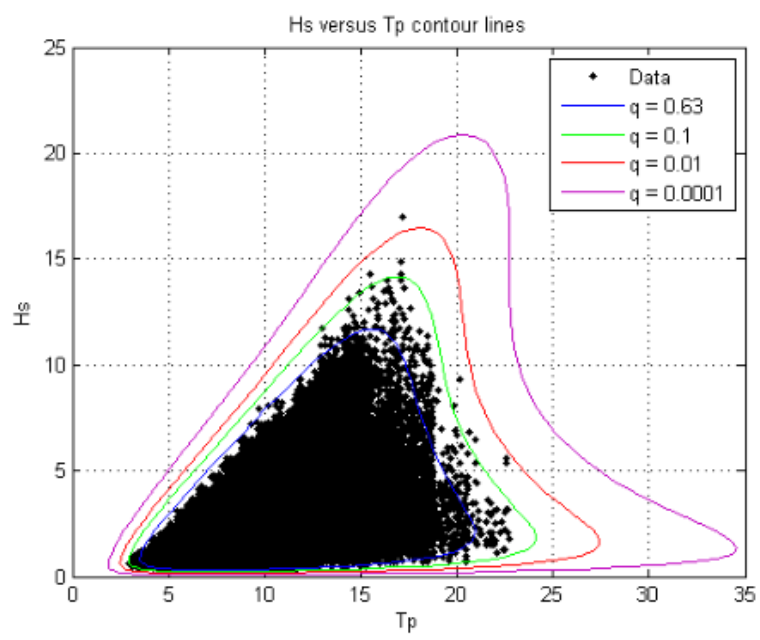

Figure 2. Environmental contour lines for Haltenbanken; $q$ is the annual prob. of exceedance. Reproduced from [20] with permission from lead author.

In paper [14], the following values are chosen to illustrate the behaviour at resonance: $F_{0}=50, k=2, \mathrm{~m}=2$ (thus, $\omega_{0}=1$ and $T_{0}=2 \pi \mathrm{s}=6.28 \mathrm{~s}$ ), $c=0.5, \omega=1.0$ (at resonance, $\left.\omega=\omega_{0}\right)$. Furthermore, the following waves are studied:

- $\quad \omega=0.5$ (at second order res.: $\omega=(1 / 2) \omega_{0}$, repr. waves with periods $T=12.56 \mathrm{~s}$ )

- $\quad \omega=0.333$ (at third order res.: $\omega=(1 / 3) \omega_{0}$, repr. waves with periods $T=18.84 \mathrm{~s}$ ), a period close to a North Sea design wave

- $\omega=0.75$, a case out of resonance. 
The phase plane diagram for a system at resonance, i.e., when $\omega=\omega_{0}=1$, exhibits a clear limit cycle, whereby all trajectories spiral towards this limit cycle. The value of the displacement would be very dependent on the value of the force, $F_{0}$, and the damping, $c$. In a traditional displacement vs. time plot, it is shown in [13] that the trajectories overlap after $30 \mathrm{~s}$.

A phase plane diagram for a system with $\omega=0.5$, i.e., at a second order resonance, also shows a clear limit cycle [13]. The trajectories overlap from approximately $t=25 \mathrm{~s}$.

\subsection{Trajectories Experiencing Apparent Negative Damping}

Some trajectories start on the inside of the limit cycle; these experience a growth in displacement and velocity amplitudes until they reach the limit cycle. These trajectories represent an increase in the motion until a limit cycle is reached. An apparent negative damping is present, feeding energy into the system.

\subsection{Drag Loading with a Current Term}

The drag loading term given in Equation (1) represents the drag loading for water waves without current. When we add a current $u_{0}$ to the system, we have the following equation:

$$
m \frac{d^{2} x}{d t^{2}}+c \frac{d x}{d t}+k x(t)=F_{0}\left(u_{0}+a \sin (\omega t)\right)\left|\left(u_{0}+a \sin (\omega t)\right)\right|
$$

Here, $u_{0}$ and $a$ are constants, $F_{0}$ represents the wave velocity amplitude. For small values of the current, $a$, limit cycles are obtained [13].

\subsection{Drag Loading on a Flexible Structure}

According to [11], for a flexible structure, the drag loading term in sinusoidal waves will take the form:

$$
F_{0}\left(b \frac{d x}{d t}+a \sin (\omega t)\right)\left|b \frac{d x}{d t}+a \sin (\omega t)\right|
$$

This means that the force will be adjusted by a term proportional to the changes in the displacement, $d x / d t$, of the structure. For small values of the forcing term $F_{0} b$, it is shown in [10] that limit cycles are obtained. Convergence towards the limit cycle is obtained after approximately $50 \mathrm{~s}$ for the parameters selected. For softer structures, the value of the term $b(d x / d t)$ is higher, and the limit cycle effect disappears [13].

A remark regarding the use of "non-linearity" should be made. Note that the squared term of the velocity component is considered to represent a non-linear component. The non-linearity due to surface effect when calculating the total force to the surface [9] would have to be implemented in a full wave loading calculation.

\section{Results; Comparison of Limit Cycles for Different Degrees of Resonance}

As discussed above, at resonances, the system stabilizes with a limit cycle behaviour for a wide range of selection of actual parameters. It is expected that wave situations with parameters in the range where limit cycles occur could be present. Figure 3 shows the limit cycles for a system described by Equation (6) at resonance (red), at second order resonance (blue), at third order resonance (magenta) and out of resonance (black); see selection of parameters below Figure 3. Figure 4 shows the displacement vs. time plot for the same trajectories. 


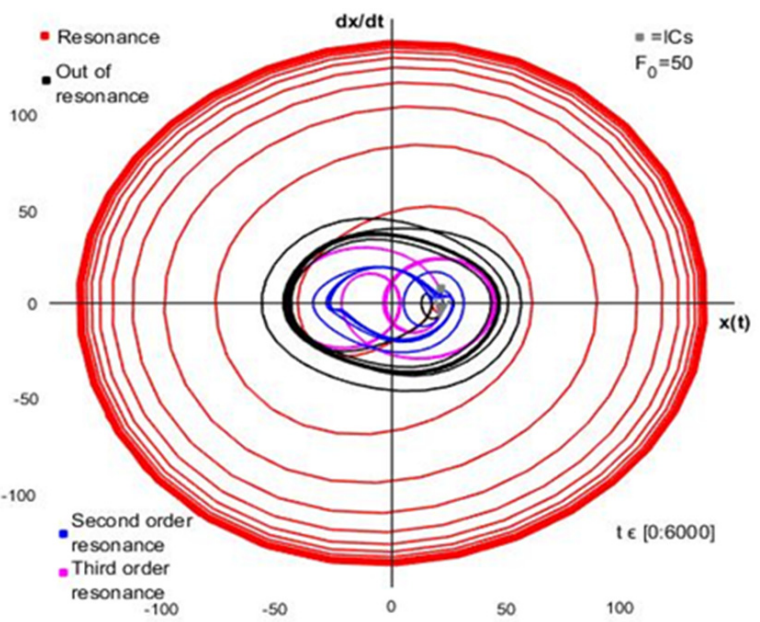

Figure 3. Systems with different degrees of resonance subjected to loading expressed by Equation (6) in the phase plane. Reproduced from [13], Published by IOP Publishing 2017.

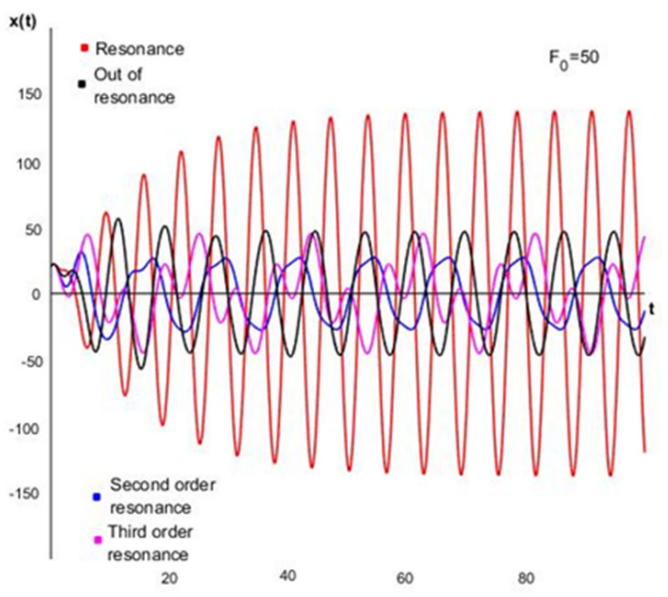

Figure 4. Position vs. time plot for systems with different degrees of resonances, subjected to loading expressed by Equation (6). Reproduced from [13], Published by IOP Publishing 2017.

So far, the same values have been used for the force amplitude, $F_{0}$, independent of the wave height. In the case of larger waves, the periods are longer (shorter wave frequencies) and the force is larger, to be reflected by a higher value of $F_{0}$. Figure 5 shows the phase plane diagram for systems at varying degrees of resonance, with the values of $F_{0}$ adjusted to represent realistic values for the non-linear forcing. The positions vs. time plot for the same systems are shown in Figure 6. Note that at resonance the wave period is $2 \pi$, at the second order resonance the wave period is $6 \pi$ and, finally, at the third order resonance the wave period is $9 \pi$. The displacement at the higher order resonances, Figure 6 , are more pronounced than at the main resonance occurring at waves with lower amplitude and higher frequencies. Thus, we conclude that waves impacting a structure with periods where higher order resonances occur can give rise to large displacements and structural velocities for systems where the loading is described by a non-linear drag component as given by the Morison equation. Although the sea is irregular, a wave situation will create incidents where resonances occur over shorter time spans, so resonances are not unfamiliar situations in an actual sea state. 


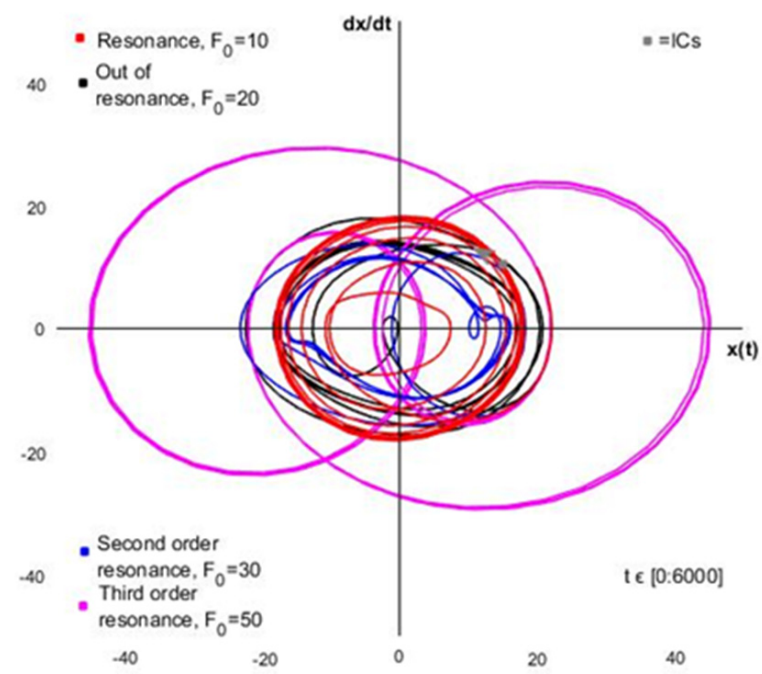

Figure 5. Phase plane diagram for systems at various degrees of resonance and realistic values of $F_{0}$, subjected to loading expressed by Equation (6). Reproduced from [13], Published by IOP Publishing 2017.

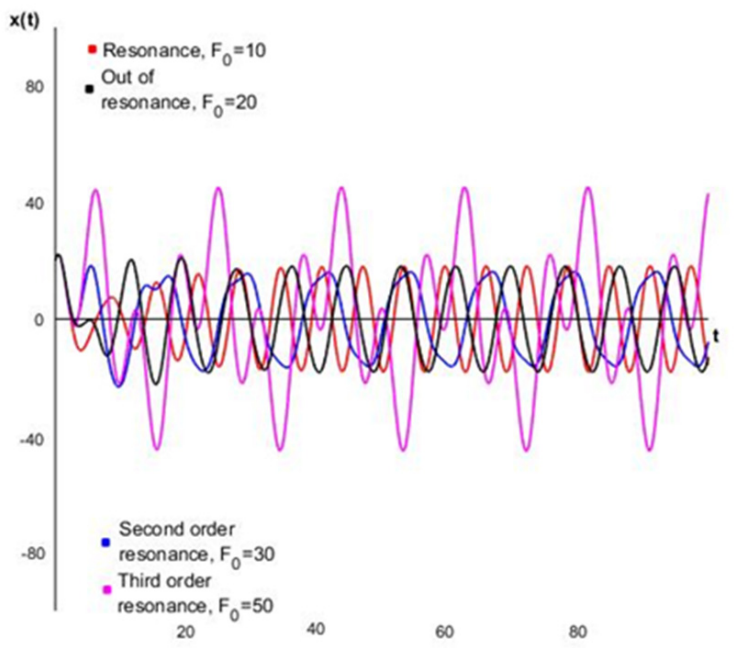

Figure 6. Position vs time plot for systems of varying degrees of resonance and realistic values of $F_{0}$, subjected to loading expressed by Equation (6). Reproduced from [13], Published by IOP Publishing 2017.

\section{Application; Large Vibrations/Ringing of Offshore Structures}

As early as 1996, Arezin et al. [21] summarized the effects of non-linear higher order dynamic effects on offshore compliant platforms. In the present paper, an attempt is made to explain an event in 1995 as a "ringing" event, resulting from the non-linear resonance between very large waves and a structure with a period $1 / 3$ of the wave period.

The interaction of the wave with the structure caused large motions of the Draugen mono-tower concrete gravity platform installed offshore in the Norwegian Sea [14]. This was actually expected, since wave tank tests, carried out at Marintek, Trondheim, Norway, during the design phase, identified that large waves could cause the platform, to be installed at $251 \mathrm{~m}$ water depth in the Norwegian Sea, to oscillate/shake heavily, as in a "ringing" mode in very large waves.

After the wave tank tests were carried out, several researchers presented explanations of the so-called ringing phenomenon. The best-known explanation is the suggestion termed "FNV" by Faltinsen et al. [22]. Liu et al. [23] analyzed the wave tank experiments conducted at Marintek, claiming that the "ringing" observed in the wave tank could be explained by diffraction theory. Their parameters, however, are different from the actual situation at Draugen during the 1995 event; the range of $k R$ values ( $k$ is the wave number and $R$ is the 
radius of the structure) in the paper are between 0.22 and 0.39 while the Draugen event took place for a rather slender structure in deep water where the combination of the radius in the waterline and the wavenumber gives a kR value closer to 0.15 . Furthermore, the Draugen situation could be termed a deep-water situation while the paper [24] considers a situation where the relation of water depth to the radius of the structure is three. An explanation that a drag-type term as suggested by the Morison equation may cause ringing-like motions for relatively slender offshore fixed structures, has been presented above, and more details are given in [14].

The column of the Draugen platform has a diameter in the range of $15 \mathrm{~m}$, so the structure is not a fully drag-dominated structure; however, for large waves, drag loading is important and represents an important contribution to the total loading. Furthermore, an unpublished study by Stansberg [24] has confirmed that a combined FNV-Morison model may explain the ringing identified by the Marintek scientists during the wave tank testing of the Draugen platform in the design phase.

With reference to the discussion in Section 4 , it is suggested that a non-linear resonance occurred between the structure and the drag loading caused by a wave component having a large amplitude and a period three times the natural period of the platform, in the range between 12 and $15 \mathrm{~s}$. The argument would have been strengthened had wave data been available; however, no measured details are available, and it is not possible for meteorologists to identify possible rogue waves from hindcast simulations.

\section{Conclusions}

Limit cycles represent the solution of a linear second order ordinary differential equation subject to non-linear drag loading. The displacements and velocities of the system will converge towards the limit cycle for initial conditions in the range of the values of the limit cycle. The linear structural damping combines with the damping caused by the drag loading to act as a negative damping in case the initial values are selected inside the limit cycle. At limit cycle, the dissipation and generation of energy are balanced.

Limit cycles are also observed when currents or structural motions (in case of a flexible structure) are included in an adjusted drag loading term.

In the case of higher order resonances, where the wave frequency, $\omega$, is a fraction $(1 / 2$ or $1 / 3$ ) of the structural frequency, $\omega_{0}$, resonances are observed which also give a limit cycle type motion. This is the case when waves with periods of $10 \mathrm{~s}$ and $15 \mathrm{~s}$, respectively, interact with a structure having a first natural period of $5 \mathrm{~s}$. Then wave energy is fed into the structural system, giving rise to large motions.

It should be noted that the forces generated by the longer period waves are larger than the forces generated by the waves with lower periods, so the effect of the resonance with high and large periodic waves is represented by large oscillatory motions. Note that, in a storm situation, the spectrum describing the sea conditions has considerable energy for high period waves.

The main contribution of the paper is to highlight the effects of resonances, in particular by linking the theory to an actual incident, the large motion of the Draugen platform in 1995.

In this paper, the focus is on large offshore structures exposed to large wave loading with long periods at higher resonance with the natural period of the structure. This gives rise to large displacements, large structural velocities and, therefore, to large accelerations and forces in the structure. Such "ringing events" could be dimensioning for structural elements.

Although not discussed in the paper, it should be noted that higher-order resonances could occur in a number of systems in electrical and mechanical engineering, as well as explaining phenomena in medicine. The heart is an ideal forcing mechanism, producing blood to move at the frequency of the heartbeat; in some situations, the heart beats faster, potentially causing resonance in the blood system. It is suggested that this should be investigated further by engineers and medical staff through close cooperation. 
Funding: This research received no external funding.

Acknowledgments: The author acknowledges the cooperation with K. Hellevik who prepared her MSc thesis under the supervision of the author; see joint paper [13]. The present paper is an extension of this joint paper with reference to the Draugen ringing event of 1995 [15].

Conflicts of Interest: The author declares no conflict of interest.

\section{References}

1. Dysthe, K.B. Note on a modification to the nonlinear Schrödinger equation for application to deep water waves. Proc. R. Soc. A 1979, 369, 105-114.

2. Trulsen, K.; Dysthe, K.B. A modified nonlinear Schrödinger equation for broader bandwidth gravity waves on deep water. Wave Motion 1996, 24, 3281-3289. [CrossRef]

3. Sand, S.E.; Ottesen-Hansen, N.E.; Jacobsen, V.; Gudmestad, O.T.; Sterndorff, M. Freak wave kinematics. In Proceedings of the NATO Advanced Research Workshop, Molde, Norway, 22-25 May 1989; Tørum, A., Gudmestad, O.T., Eds.; Kluwer Academic Press: Amsterdam, The Netherlands, 1990.

4. Haver, S. A possible freak wave event measured at the Draupner Jacket January 1 1995. In Rogue Waves; Olagnon, M., Ed.; Ifremer: Brest, France, June 2004.

5. Haver, S. Evidences of the existence of freak waves. In Rogue Waves; Ifremer: Brest, France, 2000; pp. 129-140.

6. Osborne, A.R. Breather Turbulence: Exact Spectral and Stochastic Solutions of the Nonlinear Schrödinger Equation. Fluids 2019, 4, 72. [CrossRef]

7. Morison, J.R.; O’Brien, M.P.; Johnson, J.W.; Schaaf, S.A. The force exerted by surface waves on piles. Pet. Trans. AIME 1950, 189, 149-154. [CrossRef]

8. Sarpkaya, T.; Isaacson, M. Mechanics of Wave Forces on Offshore Structures; Van Nostrand Reinhold Company: New York, NY, USA, 1981.

9. Beji, S. Applications of Morison's equation to circular cylinders of varying cross-sections and truncated forms. Ocean Eng. 2019, 187, 106156. [CrossRef]

10. Beji, S. Formulation of wave and current forces acting on a body and resistance of ships. Ocean Eng. 2020, 218, 108121. [CrossRef]

11. Gudmestad, O.T. Marine Technology and Operations. Theory and Practice; WIT Press: Southampton, UK, 2015.

12. Gudmestad, O.T.; Connor, J.J. Linearization methods and the influence of current on the nonlinear hydrodynamic drag force. Appl. Ocean Res. 1983, 5, 4. [CrossRef]

13. Hellevik, K.; Gudmestad, O.T. Limit cycle oscillations at resonances for systems subjected to nonlinear damping or external forces. IOP Conf. Series Mater. Sci. Eng. 2017, 276, 12020. [CrossRef]

14. Gudmestad, O.T. Modelling of waves for the design of offshore structures. J. Mar. Sci. Eng. 2020, 8, 293. [CrossRef]

15. Struble, R.A.; Martin, W.T. Nonlinear Differential Equations; McGraw-Hill Book Company: New York, NY, USA, 1962.

16. Hagedorn, P. Non-Linear Oscillations; Clarendon Press: Oxford, UK, 1981; p. 117.

17. Bogoliubov, N.N.; Mitropolsky, Y.A. Asymptotic Methods in the Theory of Non-Linear Oscillations; Hindustan Publ. Corp.: Delhi, India, 1961; p. 186.

18. Beuter, A.; Glass, L.; Mackey, M.C.; Titcombe, M.S. Nonlinear Dynamics in Physiology and Medicine; Springer: New York, NY, USA, 2003.

19. Guckerheimer, J.; Holmes, P. Nonlinear oscillations, Dynamical Systems, and Bifurcations of Vector Fields. In Applied Mathematical Sciences 42; Springer: New York, NY, USA, 1983; p. 67.

20. Haver, S.; Bruserud, K.; Baarholm, G.S. Environmental Contour Method: An Approximate Method for Obtaining Characteristic Response Extremes for Design Purposes. Statoil, DNV. 2012. Available online: http:/ /waveworkshop.org/13thWaves/Papers/ Sverre\%20Haver\%20_\%20Wave\%20Hindcast $\% 20 \% 20$ Forecast $\% 20 \% 20$ Environmental\%20contour\%20method.pdf (accessed on 10 April 2020).

21. Arezin, R.; Bar-Avi, P.; Benaroya, H. Dynamic response of compliant offshore structures-Review. J. Aerosp. Eng. 1996, 9, 114-131. [CrossRef]

22. Faltinsen, O.M.; Newman, J.N.; Vinje, T. Nonlinear wave loads on a slender vertical cylinder. J. Fluid Mech. 1995, $289,179-198$. [CrossRef]

23. Liu, Y.; Xue, M.; Yue, D.K.P. Computations of fully nonlinear three-dimensional wave-wave and wave-body interactions, Part 2: Nonlinear waves and forces on a body. J. Fluid Mech. 2001, 438, 41-66. [CrossRef]

24. Stansberg, C.T. Comparing simple models for prediction of ringing loads and responses. In Sintef Report 580133.00.01; SINTEF: Trondheim, Norway, 2009. 